\title{
Precision Peg-in-Hole Assembly Strategy Using Force-Guided Robot
}

\author{
Yin $\mathrm{Xu}^{\mathrm{a}}$, Yue Hu ${ }^{\mathrm{b}}$, Lei Hu${ }^{\mathrm{c}}$ \\ BeiHang University, Beijing 100191, China \\ axuying@buaa.edu.cn, bspace_111@126.com, chulei9971@sina.com
}

Keywords: Precision assembly, circle shaft and hole, robotic assembly, force-guided.

\begin{abstract}
Position error is inevitable in robotic precision assembly task, so force sensor is needed to get information of the environment and guide the motion of robot. In order to describe assembly task clearly, we divided it in three phases: 1 .Move to a certain position; 2 .Move the shaft to the exactly top of the hole; 3. Put the shaft down in the hole. Phase 1 is easy because the position of the hole and shaft is already known. In Phase2, we divide the contact area in sixteen regions, and detect the regions using KD-tree method. After detecting the regions, a coordinate search path is followed in finding the position of the hole. In phase3, we fine adjust the shaft on the X-Y plane according to the value of $F_{x}$ and $F_{y}$, and put the shaft down in the hole. In order to validate the strategy, experiments are conducted on a Cartesian coordinate robot. Multiple position error is considered and the strategy perform well.
\end{abstract}

\section{Introduction}

Many robotic assembly strategies are raised in laboratory environment, but in real world most of the investigation is limited to automotive industry and electronic industry. So it is still important to design available strategy in robotic assembly. In robotic assembly, robot need to contact the environment, and adjust pose according to the environment. So the assembly strategies can be divided into two parts: passive assembly strategy and active assembly strategy.

Passive strategy is building a RCC compliant wrist with specific stiffness matrix based on the specific assembly environment, or building the VRCC wrist, which is considered improved RCC.

As to active strategy, environmental information must be obtain by some sensor, robot execute the action according to the gained information to complete assembly operations. Visual sensor and force sensor is often applied to access to environmental information. Due to the variable illumination, grease and other complex factors in factory, application of visual system is difficult. So force sensor is desired in identification of environmental information for active strategy. Compared to passive strategy, although expensive force sensor is required, but this method is more flexible, safe and under control, and it is more easy to adapt to flexible transfer line.

Many previous studies are done in order to achieve active assembly strategy. This paper confined the assembly object in single round peg-in-hole assembling, square shaft and hole or multi shaft and hole in one component is discussed in paper [1][2][3][4], if the shape of component is no single round, you can read those paper, we will not discuss those questions here. In [5] Hidden Markov model is used to identify five different contact state, a dynamic model is established based on compliance contact mechanics in [6]. In [7, 8, 9], concrete implementation methods and strategies are raised. The strategies suggest above need to change the pose of end effector, and so the robot must have at least six degree of freedom. If using SCARA robot, you will need to install an active compliance wrist. In the actual assembly process, the deviation of pose between shaft and hole can be adjust once to meet assembly requirement. In [10] the function of force/torch and contact position is given under detail derivation, but when we use the function, the contact position and calculated result are quite different. Because although the small errors of attitude will not affect the assembly process, but it will lead the calculation has a large deviation. The other reason is the force/torch number acquired is not the reality value. So dividing the contact position to several different states, using pattern recognition technique to identify those states and designing high efficiency search strategy to accomplish the task. In [11] 
the contact position is divided into equal 48 regions and a pattern recognition method is given to identify them. But the method it raised is too simple and the algorithm details is not given. In [12, 13] Gaussian mixture distribution method is applied in pattern recognition. Paper [12] also designed a spiral search path to find the position of the hole. But it takes a long time when used in precise assembly, because the spanning distance $b$ will be very small.

The KD-Tree model is used to identify the different state and then a coordinate search path method is used to determine the position of the hole. The rest of the article is organized as follows; in section 2 force-guided robotic peg-in-hole assembly task is described. Section 3 gives the proposed assembly algorithm. Experimental validation and concluding remarks are discussed in section 4.

\section{Task Description}

If using SCARA robot or Cartesian coordinate robot, you will need to install an active compliance wrist. In the actual assembly process, the deviation of pose between shaft and hole can be adjust once to meet assembly requirement. Therefore we only discuss the assembly strategy when pose deviation is small enough.

The entire assembly process is divided into three phases; Move to a certain position; 2.Move the shaft to the exactly top of the hole; 3 . Put the shaft down in the hole. Phasel is easy because the position of the hole and shaft is already known. In Phase2, we divide the contact area in sixteen regions, and detect the regions using KD-Tree method. After detecting the regions, a coordinate search path is followed in finding the position of the hole. In phase3, we fine adjust the shaft on the $\mathrm{X}-\mathrm{Y}$ plane according to the value of $F_{x}$ and $F_{y}$, and put the shaft down in the hole.

The process is shown in Fig.1, in phase 1 the shaft move from position (a) to (b); in phase 2 the shaft move from position (b) to (c); and finally the shaft move down from (c) to (d), the entire process is completed.

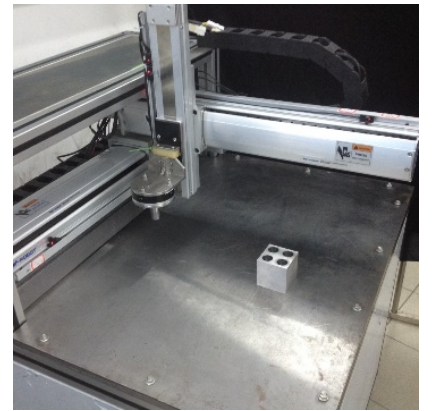

(a) Initial Position

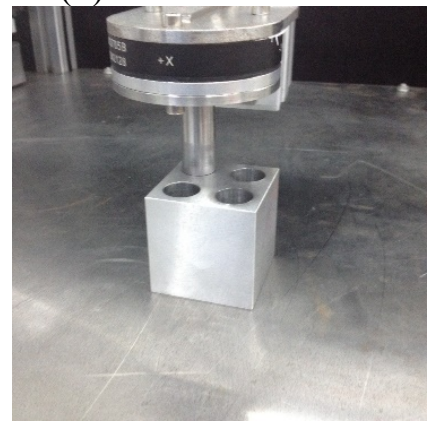

(c) Aligned with the hole

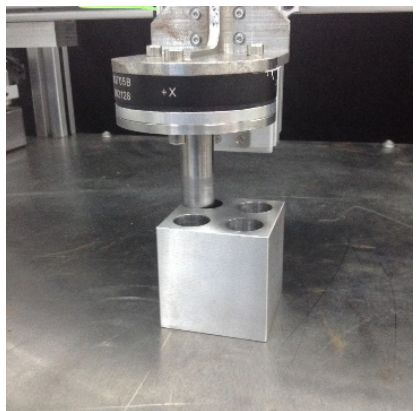

(b) contact the hole

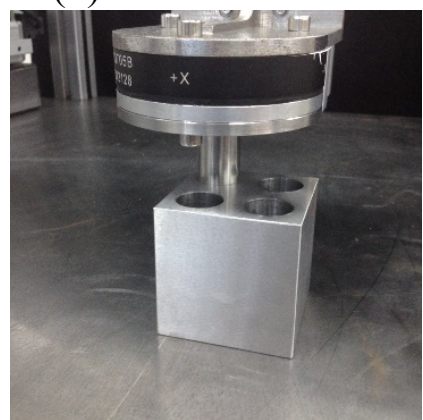

(d) Inserted in the hole Fig. 1 Different nodes in assembly process

\section{Assembly Strategy}

As different contact position produce different force/torch value, for instance, when the shaft is exactly on the top of hole, the contact force/torch will be nearly zero, so pattern recognition method can be used to detect different states. A detail must be pointed out here, when the shaft has no contact with the hole, the force/torch value need to set to zero in the program. 


\subsection{Detect Different Contact States}

The force coordinate system and world coordinate system is shown in Fig.2, D reference to the center contact point of contact area, $d$ reference to clearance distance, all potential $\mathrm{D}$ is within the clearance distance, and we call it deviation area. Then the deviation area is divided into sixteen area, plus the state when the shaft is aligned with the hole, there are seventeen different states. Many kinds of pattern recognition method can be used, including KD-Tree, Bayesian classifier, Hidden Markov model, Gaussian mixture distributions and so on. KD-Tree method is used here, because it is easy to understand and the data may not fit Gaussian distribution. The identification model of KD-Tree in this application is shown in Fig.3. First step, the state when the shaft is aligned with the hole is detected. Then when the shaft is contacting with the hole component, different contact area will be detected.
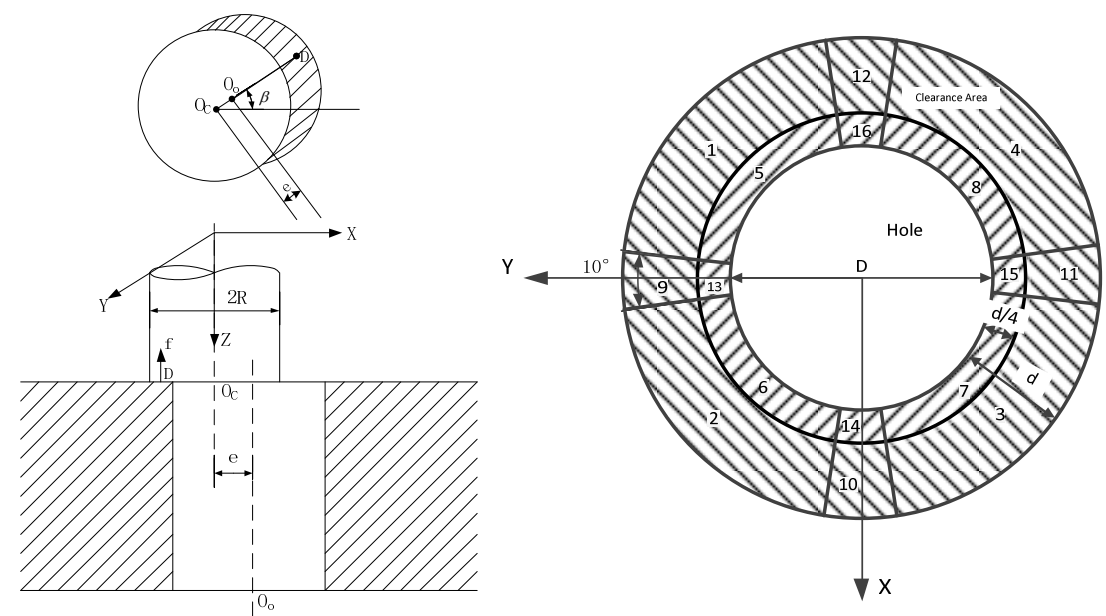

Fig.2 Clearance and different clearance area

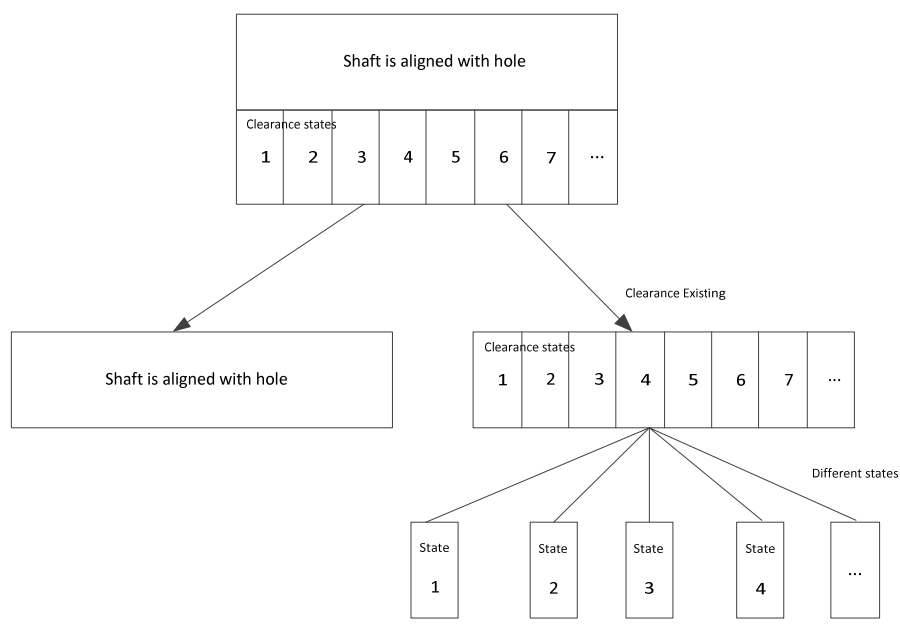

Fig.3 Model of recognition of different states

Find the Principle Component

Six-axis force senor can capture Cartesian force signals and Cartesian torch signals, so force vector $\left(F_{x}, F_{y}, F_{z}, M_{x}, M_{y}, M_{z}\right)$ can be used to detect different states. The force vector has six components, but not all components contribute equal to distinguish the states. It is clearly that $F_{z}$ change significantly when the shaft move from states $1 \sim 16$ to state 17 , while relationship between states and $M_{z}$ is weak. But the value will fluctuate because of noise signal. So $\left(F_{x}, F_{y}, F_{z}, M_{x}, M_{y}\right)$ is chosen as feature vector to detect different states.

\section{Measuring Sampling Data}

Move the shaft to the states above, for every state a certain number of feature vectors are captured. All this vectors form a feature space. Thus, for each state there is a feature space. Each feature space has the same number of vectors, $\mathrm{N}$ stands for the number. 
Calculating Weight of Component of the Feature Vector

As said above, each element of the selected five-dimensional contribute different to identify different states. We can give different weight factor to present the difference. The method to calculate the weight factor is as follow.

As we all know, covariance can stands for dependence of variables, so we can use covariance value as weight value. In the deviation area, contact point $\mathrm{D}$ can have deviation in both $\mathrm{X}$ and $\mathrm{Y}$ direction. Move the contact point $\mathrm{D}$ to the point only has deviation in $\mathrm{X}$ direction, and every $0.5 \mathrm{~mm}$ within deviation $-4 \mathrm{~mm}$ to $4 \mathrm{~mm}$. Then calculate the covariance of $\mathrm{X}$ and each component of feature vector. Do the same in Y direction. Chose the bigger one as the weight value of different component, record as $\left(w_{1}, w_{2}, w_{3}, w_{4}, w_{5}\right)$.

\section{Recognition Method}

If a vector $\left(F_{x}, F_{y}, F_{z}, M_{x}, M_{y}\right)$ is given, we can tell which state produce the vector. Calculate the sum of distance between the vector and every feature vector in feature space, then compare the sum of distance. The lowest one stands for it belong to the feature space. The formula is given as follows, here e stands for the sum of distance.

$$
\begin{aligned}
& e_{j}=\sum_{i=1}^{N} \sqrt{w_{1}\left(F_{x}-F_{x_{i}}\right)^{2}+w_{2}\left(F_{y}-F_{y_{i}}\right)^{2}+w_{3}\left(F_{z}-F_{z_{i}}\right)^{2}+w_{4}\left(M_{x}-M_{x_{i}}\right)^{2}+w_{5}\left(M_{y}-M_{y_{i}}\right)^{2}} \\
& j=\operatorname{Min}\left(e_{j} ; j\right)
\end{aligned}
$$

\subsection{Align the shaft with the hole}

When the fit tolerance between shaft and hole is large, the spiral search path may be useful. But if the fit tolerance is small, the parameter b in spiral search path [10] will be small, and the time cost will be much more. The search method is given as follows.

As shown in Fig.2, region 9,11,13,15 have little deviation in $\mathrm{X}$ direction, region 10,12,14,16 have little deviation in $\mathrm{Y}$ direction. In fact, we find that when deviation value is fixed in one direction, for example $\mathrm{X}$, and can move in another direction $\mathrm{Y}$, the lowest value exist when the deviation is lower in Y. The feature can be detected easily. So the strategy is when the contact point is not in the above area, first move the shaft along $\mathrm{X}$ direction to region 9,11,13,15, then move along $\mathrm{Y}$ direction to region $5,6,7,8,13,14,15,16$. Repeat this operation and gradually reduce the step size to a minimum step size, until approaching state 17.

The proposed operation process is clearly shown as follows:

Detect the contact state;

Set the step size as $S=d / 2$, if adjust number increased by two, then $S=S / 2$, if $S<0.1, S=0.1$;

If state $1 \sim 4$ is detected, move the shaft in $\mathrm{X}$ direction by step size $\mathrm{S}$, go to step a;

If state $5 \sim 8$ is detected, move the shaft in $\mathrm{X}$ direction by step size $\mathrm{S}$, add the adjust number by one, go to step a;

If state 9, 11 is detected, move the shaft in Y direction by step S, go to step a;

If state 13, 15 is detected, move the shaft in Y direction by step S, add the adjust number by one, go to step a;

If state 10,12 is detected, move the shaft in $\mathrm{X}$ direction by step size $\mathrm{S}$, go to step a;

If state 14,16 is detected, move the shaft in $\mathrm{X}$ direction by step size $\mathrm{S}$, add the adjust number by one, go to step a;

If state 17 is detected, the shaft is aligned with the hole.

\subsection{Insert the Shaft Down in the Hole}

After the hole is reached, now we can move the shaft down in $\mathrm{Z}$ direction i.e. put the shaft down in the hole. Although pose deviation is small enough in this strategy, jamming will exist when put the shaft down. As it is shown in Fig.4, this is caused by the small pose deviation. But we do not need to adjust the pose to fulfil this step, move the position of shaft slightly in X-Y plane can solve the problem. 
When the shaft is in the hole, and jamming state is existing, the value of $F_{x}, F_{y}$ will be increase significantly. Now we just move the shaft along the direction of $F_{x}, F_{y}$ until the value of $F_{x}, F_{y}$ reduce to the threshold value you set.

For instance, if both $F_{x}$ and $F_{y}$ surpass the threshold value, now first move the shaft along $F_{x}$ or $F_{y}$ until $F_{x}$ or $F_{y}$ reach its minimum number, then move the shaft along $F_{y}$ or $F_{x}$ until $F_{y}$ or $F_{x}$ reach its minimum number, repeat this operation until insert depth is enough.

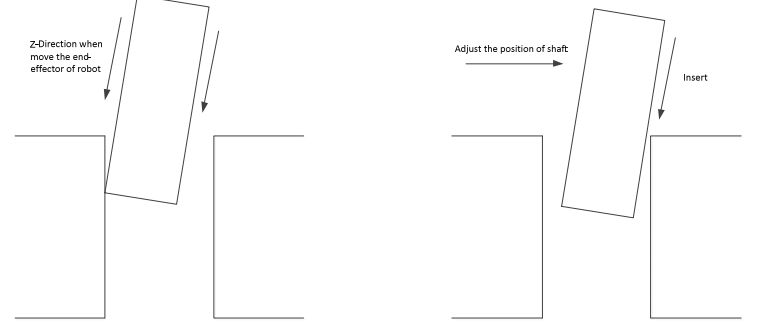

Fig.4 Strategy when come across jamming condition

\subsection{The Proposed Assembly Algorithm}

As the recognition model of different contact states is developed, then one can use them in finding the accurate hole position in coordinate search path explained above. After that one can inserting the shaft down in the hole by position adjust according to force value? The whole process can be summarized by the following algorithm:

According to the relative position of the shaft and hole, move the shaft to the initial position. Then move down along $Z$ direction until contact force $F_{z}$ reach the set value;

Align shaft with hole using strategy given above;

Move shaft down along $\mathrm{Z}$ direction;

If $F_{z}$ beyond threshold value, check $F_{x}$ and $F_{y}$, move along $F_{x}$ or $F_{y}$ direction until $F_{x}$ or $F_{y}$ reach the minimum number. Then check $F_{z}$. If $F_{z}$ within the threshold value, move to step c, else move to step e;

Move along the direction of $F_{y}$ or $F_{x}$ until $F_{y}$ or $F_{x}$ reach the minimum number. Then check $F_{z}$. If $F_{z}$ within the threshold value, move to step c, else move to step d;

If insertion length reach set value, the process is completed; 


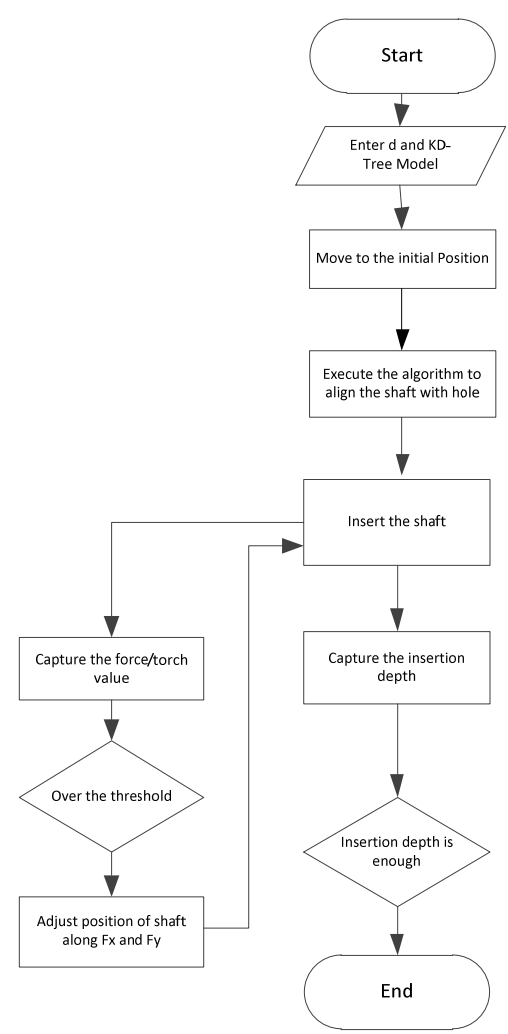

Fig.5 The flow chart of the proposed assembly algorithm

\section{Experimental Validation and Conclusion}

\subsection{Experimental Validation}

In order to verify the validity of the assembly strategy, a platform was built. The platform is composed of a Cartesian coordinate robot and a SRI-M3705B six-axis force sensor which can capture the Cartesian coordinate force/torch value. Personal Computer and motion control card is used to control the motion of force-guided robot. The feature of the PC is of Inter (R) Core (TM) i5-2300 CPU with $2.8 \mathrm{GHz}$ speed and $4 \mathrm{~GB}$ RAM running under a Windows 7 environment. The programming is done through a $\mathrm{C}++$ platform.

The design radius of shaft and hole is $20 \mathrm{~mm}$, clearance fit, and fit clearance is $0.02 \mathrm{~mm}$, the insertion depth is about $30 \mathrm{~mm}$. The test equipment is shown in Fig. 1. The parameter d shown in Fig.2 is set to $4 \mathrm{~mm}$, the contact value $F_{2}$ is set to $5 \mathrm{~N}$, the threshold value in Phase 3 is set to $40 \mathrm{~N}$, and the threshold value of $F_{X}$ and $F_{Y}$ is set to $2 \mathrm{~N}$.

We set the initial position in phase 1 in different contact states shown in Fig.2. Compare to spiral search path, the Cartesian coordinate search method cost less time. It can complete assembly in 3 seconds.

\subsection{Conclusion}

When the algorithm is running, most the time is cost in recognize different states. If the number of states increase, the computational time increase more. If the function of contact position and force/torch value can be find by statistics analysis, the whole assembly process can be more easy and the time it cost will be much less.

\section{Acknowledgement}

This work is supported by National Science and Technology Major Project of the Ministry of Science and Technology of China (Grant NO 2014ZX04013011-02). 


\section{References}

[1] Strip D R. Insertions using geometric analysis and hybrid force-position control: method and analysis[C]// Robotics and Automation, 1988. Proceedings, 1988 IEEE International Conference on IEEE, 1988:1744-1751 vol.3.

[2] Chhatpar S R, Branicky M S. Search Strategies for Peg-in-Hole Assemblies with Position Uncertainty[C]// Intelligent Robots and Systems, 2001. Proceedings. 2001 IEEE/RSJ International Conference on IEEE, 2001:1465-1470 vol.3.

[3] Kim Y L, Kim B S, Song J B. Hole detection algorithm for square peg-in-hole using force-based shape recognition[C]// Automation Science and Engineering (CASE), 2012 IEEE International Conference on IEEE, 2012:1074-1079.

[4] FEI Yan-qiong, ZHAO Xi-fang. Modeling of Contact Force in Robot Multiple Peg-in-Hole in Three Dimensions [J]//Journal of Shanghai Jiaotong University, 2003, 37:703-705. DOI:doi:10. 3321/j. issn: 1006-2467.2003.05.019.

[5] Itabashi K, Hirana K, Suzuki T, et al. Modelling and realization of the peg-in-hole task based on hidden Markov model[C]// Robotics and Automation, 1998. Proceedings. 1998 IEEE International Conference on IEEE, 1998:1142 - 1147.

[6] Xia Y, Yin Y, Chen Z. Dynamic analysis for peg-in-hole assembly with contact deformation [J]. International Journal of Advanced Manufacturing Technology, 2006, 30(1-2):118-128.

[7] Leong K W, Usubamatov R. Analyses of peg-hole jamming in automatic assembly machines [J]. Assembly Automation, 2011, 31(4):358-362.

[8] Yamashita T, Godler I, Takahashi Y, et al. Peg-and-hole task by robot with force sensor: Simulation and experiment[C]// Industrial Electronics, Control and Instrumentation, 1991. Proceedings. IECON '91. 1991 International Conference on IEEE, 1991:980-985 vol.2.

[9] Xia Yanchun, Yin Yuehong, Huo Hua. Study on Methodology of Setting Active Compliance Center for Peg-in-Hole Assembly Task[J]. Chinese Journal of Mechanical Engineering, 2004, 15:1015-1017. DOI:doi:10.3321/j.issn:1004-132X.2004.11.020.

[10] Li Xiaobing. Study of Robot Active Assembly Method for Round Peg-hole[J]. Journal of University of Electronic Science and Technology of China, 2001, 30:49-52. DOI:doi:10. 3969/j.issn. 1001-0548.2001.01.013.

[11] Li Wenying, $\mathrm{Hu}$ Wenfeng. An Active Compliance Strategy for Robotic Insertion Assembly_Pattern Recognition Methhod[J]. High Technology Letters, 1994:16-20.

[12] Jasim I F, Plapper P W, Voos H. Position Identification in Force-Guided Robotic Peg-in-Hole Assembly Tasks[J]. Procedia Cirp, 2014, 22(23):217-222.

[13] Jasim I F, Plapper P W. Contact-State Recognition of Compliant Motion Robots Using Expectation Maximization-Based Gaussian Mixtures[C]// ISR/ Robotic 2014; 41st International Symposium on Robotics; Proceedings of VDE, 2014:1-8. 